\title{
Effect of alternate day collection on semen quality of Asian elephants (Elephas maximus) with poor initial fresh semen quality
}

P. Imrat ${ }^{1}$, S. Mahasawangkul ${ }^{2}$, C. Thitaram ${ }^{3}$, P. Suthanmapinanth ${ }^{4}$, K. Kornkaewrat ${ }^{4}$, P. Sombutputorn ${ }^{2}$, S. Jansittiwate ${ }^{2}$, N. Thongtip ${ }^{4}$, A. Pinyopummin 4 , B. Colenbrander ${ }^{1}$, W.V. Holt ${ }^{5}$ and T.A.E. Stout ${ }^{1,6}$

'Department of Equine Sciences, Faculty of Veterinary Medicine, Utrecht University, Yalelaan 114, 3584 CM Utrecht, The Netherlands.

${ }^{2}$ National Elephant Institute, Forest Industry Organization, Lampang, 52190, Thailand.

${ }^{3}$ Faculty of Veterinary Medicine, Chiang Mai University, Chiang Mai, 50100, Thailand.

${ }^{4}$ Faculty of Veterinary Medicine, Kasetsart University, Nakhonpathom, 73140, Thailand.

5Institute of Zoology, Zoological Society of London, Regent's Park, London, NW1 4RY, UK.

${ }^{6}$ Department of Production Animal Studies, University of Pretoria, Private Bag X20 Hatfield, Pretoria 0028, South Africa

Contact details of corresponding author:

Podjana Imrat

E-mail address: p imrat@yahoo.com 


\section{Abstract}

In captivity, male Asian elephants often yield poor quality semen after transrectal manually assisted semen collection; however, the reasons for the disappointing semen quality are not clear. Here we test the hypothesis that accumulation of senescent spermatozoa is a contributory factor, and that semen quality can therefore be improved by more frequent ejaculation. To this end we investigated the effect of collecting semen five times on alternate days, after a long period of sexual rest, on semen quality in Asian elephants known to deliver poor semen during infrequent single collections. All eight bulls initially displayed a high incidence of detached sperm heads and low percentages of motile (close to $0 \%$ ) spermatozoa. After semen collection on alternate days, the percentages of detached sperm heads, and head and mid-piece abnormalities, were reduced significantly $(\mathrm{p}<0.05)$. In particular, one bull showed markedly improved sperm motility (increased from $0 \%$ to $60 \%$ ) and membrane integrity (increased from $5 \%$ to $75 \%)$. In addition, advancing age significantly $(p<0.01)$ correlated with lower percentages of sperm with intact membranes and a higher frequency of detached sperm heads. In contrast to sperm accumulation problems in other species, a small ampullary diameter correlated significantly $(p<0.05)$ with reduced semen quality.

Keywords: poor semen quality, frequent semen collection, ampullae, Asian elephant 


\section{Introduction}

The Asian elephant is considered to be under significant threat of extinction and is included in Appendix 1 of the Convention for International Trade in Endangered Species of Wild Fauna and Flora (CITES). Captive populations have therefore been established in zoos and camps for the conservation of the species (Brown, 2000); however, these captive populations have been affected by low birth rates and are considered to be at risk of inbreeding because of the combined threats of a high rate of reproductive pathology among an ageing female population, the small numbers of bulls used for breeding, and fragmentation of the breeding pools (Thitaram, 2012). Artificial insemination (Al) has been developed as a potentially useful tool for genetic and breeding management in captivity (Schmitt et al., 2001; Thongtip et al., 2009). Unfortunately, male Asian elephants in captivity typically have a high proportion of defective or immotile spermatozoa in their ejaculates (Saragusty et al., 2009). The reasons for the poor semen quality in a high proportion of Asian elephants is still not clear (Thongtip et al., 2008b).

In bulls and stallions, one of the causes of poor semen quality in animals that are not used for frequent mating or semen collection is the accumulation of senescent spermatozoa in the ampullae, vas deferentia and cauda epididymides. Senescent semen is characterized by a high percentage of dead spermatozoa and a high percentage of spermatozoa with detached heads (Barth, 2007). In farm animals, the elimination of non-ejaculated spermatozoa mostly occurs by intermittent emission into the urethra followed by evacuation during urination. Any non-ejaculated spermatozoa that are not eliminated into the urethra accumulate in the sperm storage areas (cauda epididymides, ampullae) and gradually degenerate (Garner and Hafez, 2000). In stallions and bulls, the senescent spermatozoa often accumulate in the ampullae of the deferent ducts, resulting in an increase in the diameter of the lumen of the ampullae; the negative effects of sperm accumulation on semen quality can, however, generally be rectified by a period of frequent, repeated ejaculation. Semen of domestic (cattle) bulls affected by accumulation of senescent spermatozoa can be improved by collecting semen three times daily for up to one week (Barth, 2007). For similar reasons, the French National Stud suggests collecting semen daily for $5 \mathrm{~d}$ prior to evaluating new stallions entering the stud for semen quality. Even stallions that have already have been on stud duty may require more than twice daily semen collection 
for 2 - $10 d$ to maximize semen quality after a period of sexual rest, although the exact frequency of semen collection has to be adjusted to each individual (Magistrini, 2000; Pycock, 2008). Male Asian elephants in captivity are usually housed individually and rarely have a chance to breed a female. Indeed, most have never had the opportunity to naturally mate a cow. Even though bull elephants will intermittently discharge semen at night, it therefore seems reasonable to suggest that sperm accumulation and degeneration may occur during the considerable periods of sexual rest that most elephants experience, as it does in other species. Male age also influences various aspects of reproductive performance, including semen quality. Poor reproductive capacity is often associated with very young, immature males (Parthasarathy and Nair, 2006), while aging males also show a decline in semen quality (Kidd et al., 2001) and reproductive tract function in general (Elzanaty, 2007).

The present study was undertaken primarily to determine whether increasing the frequency of semen collection in Asian elephants with poor semen quality could elicit improvements. The objectives of the study were to investigate changes in semen quality when semen was collected on alternate days over a $10 \mathrm{~d}$ period, and to compare the changes between bulls in different age groups. We expected to see improvements in semen quality with successive ejaculates. Because dilation of the ampullae is associated with sperm accumulation in domestic bulls and stallions (Barth, 2007), the diameter of the ampullae was also estimated using transrectal ultrasonography and its association with semen quality was investigated.

\section{Materials and Methods}

\section{Chemicals and experimental location}

All reagents were purchased from Sigma Chemical Company (Sigma, St. Louis, MO, USA) unless otherwise stated. Semen collection and evaluation were performed the National Elephant Institute (NEI), Lampang, Thailand (latitude $18^{\circ} 21.60^{\prime} \mathrm{N}$ and longitude 99¹4.92'E).

\section{Semen collection and evaluation}

Semen was collected from the elephant bulls by transrectal massage of the ampullae, as described by Schmitt and Hildebrandt (Schmitt and Hildebrandt, 1998). Immediately after collection, each ejaculate was evaluated for volume, $\mathrm{pH}$, sperm concentration, 
and the percentages of motile, membrane intact, morphologically normal and abnormal spermatozoa.

A haemocytometer was used for sperm concentration assessment (Bane, 1952), and the percentage of motile spermatozoa was estimated subjectively using a phasecontrast microscope (Olympus model CHS, CH-2 series; Olympus, Tokyo, Japan) at a magnification of $x 200$ to examine a standardized $10 \mu \mathrm{L}$ drop of semen pipetted onto a pre-warmed $\left(3^{\circ} \mathrm{O}\right)$ glass slide and covered with a cover-slip. Sperm membrane integrity was evaluated using eosin-nigrosin staining (Bjorndahl et al., 2003). Two hundred spermatozoa per sample were examined for membrane integrity (unstained = intact membrane; stained = damaged membrane). Sperm morphology was assessed using William's stain (carbol-fuchsin) as described by Sarder (2008). Smears of fresh semen were air-dried and fixed in absolute alcohol for $3 \mathrm{~min}$ and then stained with William's stain for 5 min. After staining, the slides were washed in running tap water, dried and examined using bright-field light microscopy (Olympus model $\mathrm{CHS}, \mathrm{CH}-2$ series; Olympus, Tokyo, Japan) at a magnification of $\mathrm{x} 1000$. The extremely poor quality semen recovered from the bulls during initial collections contained such high percentages of spermatozoa with detached heads, that the other categories of morphological abnormalities became extremely small numerically. To better examine changes in these other sperm defects, detached heads were treated separately from the other morphological parameters in subsequent analysis. Two hundred spermatozoa per sample were examined for detached or attached heads (intact) and two hundred intact spermatozoa per sample were examined for other head, mid-piece or tail abnormalities, or classified as having normal morphology. If a spermatozoon with an attached head had multiple defects, all defects were recorded separately. The percentage of morphologically normal spermatozoa was calculated as (Total spermatozoa with attached head (\%) x intact spermatozoa with normal morphology (\%))/100.

\section{Experimental design}

\section{Experiment 1: Effect of alternate day semen collection}

Bulls that had a history of routinely producing semen with less than $5 \%$ motile spermatozoa were used in this experiment. Following a period of sexual rest of at least one year, semen was collected from eight bulls (A1, A2, A3, B1, B2, B3, B4 and B5) 
on alternate days for $10 \mathrm{~d}$ (5 ejaculates per bull in total) during November 2009 January 2010. The ages of $A 1, A 2, A 3, B 1, B 2, B 3, B 4$ and B5 were 47, 54, 59, 37, $36,34,21$ and 22 y, respectively. Semen quality was evaluated immediately after the collection of each ejaculate. The correlation between age and semen quality was analyzed over all ejaculates; i.e., 40 ejaculates in total (5 ejaculates from 8 bulls).

\section{Experiment 2: significance of ampullar diameter}

Two sires from the NEI (Bull1 and Bull2) and four bulls (A2, A3, B4 and B5) from experiment 1 were used in this experiment. They were not used for natural breeding and semen collection for at least one month prior to this study. In July 2010, their left and right ampullae were examined by transrectal ultrasonography (Hildebrandt et al., 2000) using a SonoSite Vet 180 Plus ultrasound scanner equipped with a C11/7-4 transducer (SonoSite Inc., San Francisco, CA, USA). The maximum diameters of cross-sectional sonograms were measured for both ampullae. After ultrasonographic examination, semen was collected and immediately evaluated for quality for all six bulls.

\section{Statistical analysis}

Statistical analyses were performed using SPSS 17.0 for Windows (SPSS Inc., Chicago, IL, USA). All semen quality parameters were checked for normality of distribution using the Kolmogorov-Smirnov test. For semen quality comparisons between the first and last ejaculates in experiment 1, Paired $t$-tests were used. Percentages of motile and membrane intact spermatozoa, and of attached head spermatozoa with head or tail abnormalities were square root transformed before being analyzed using Paired $t$-tests. Spearman's rank correlation was used to analyze the correlation between age and semen quality in experiment 1 , and the correlation between ampullar diameter and semen quality and age in experiment 2.

\section{Results}

\section{Experiment 1: Effect of alternate day semen collection}

The average value of all semen parameters, including semen volume, concentration and $\mathrm{pH}$, the percentages of motile, membrane intact and morphologically normal spermatozoa, the percentage of spermatozoa with detached heads and the 
percentages of intact spermatozoa with normal morphology or with head, mid-piece and/or tail abnormalities, were compared between the first and the last ejaculate of the alternate day semen collection protocol (Table 1). Semen $\mathrm{pH}$, the percentage of spermatozoa with detached heads, the percentages of intact sperm with abnormal heads and or mid-pieces and the percentage of morphologically normal spermatozoa differed significantly $(p<0.05)$ between the first and last ejaculates. While semen $\mathrm{pH}$ increased from neutral to alkaline, the percentage of spermatozoa with detached heads and the percentages of intact spermatozoa with head abnormalities and midpiece abnormalities decreased after alternate day semen collection. Accordingly, the percentage of morphologically normal spermatozoa increased significantly after alternate day semen collection. In bull B1 in particular, the percentage of motile and membrane intact spermatozoa improved dramatically after alternate day semen collection (Figure 1), while the percentage of spermatozoa with detached heads decreased and semen $\mathrm{pH}$ changed from acidic to neutral. From the overall results for semen quality for the bulls in this experiment, additional significant correlations between age and some semen quality parameters were evident. The percentage of membrane intact spermatozoa $(r=-0.734, p<0.01)$ correlated negatively with age, while the percentage of spermatozoa with detached heads $(r=0.608, p<0.01)$ correlated positively with age (Figure 2).

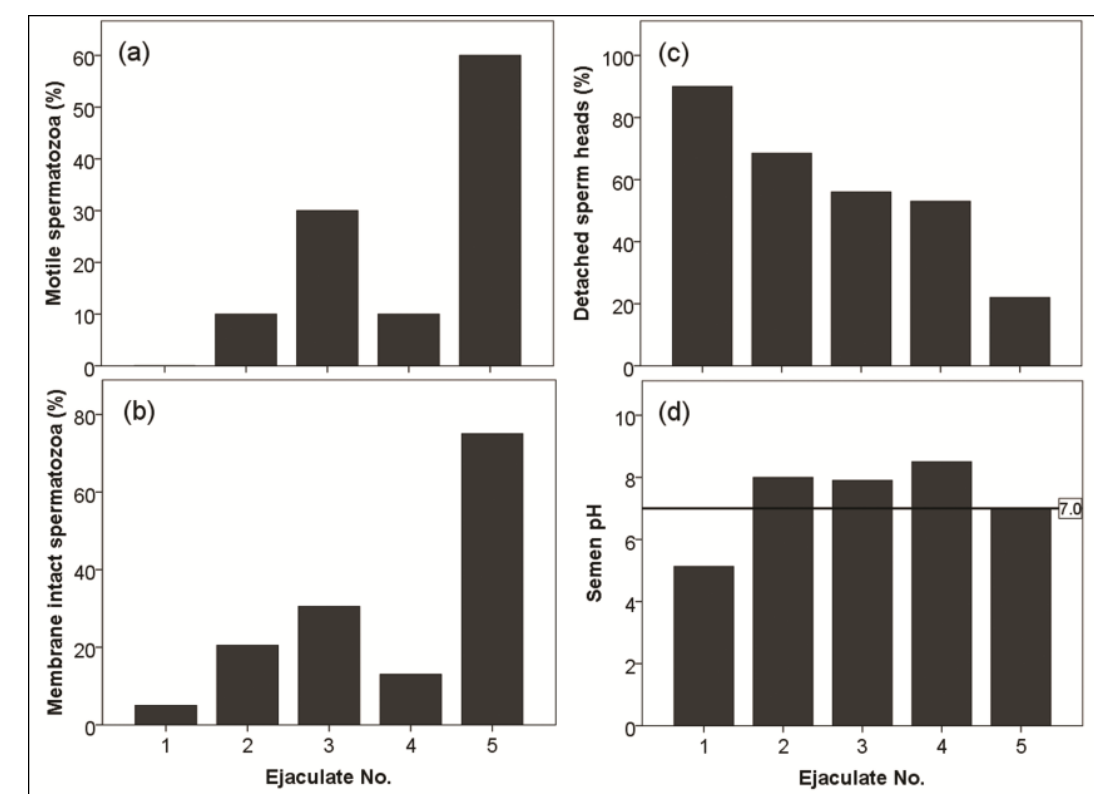

Figure 1: Changes in semen quality during alternate day semen collection (first to fifth ejaculates) for Asian elephant bull B1 ( $n=1$, a young mature adult); marked changes were observed for (a) sperm motility (\%), (b) sperm membrane integrity (\%), (c) detached sperm heads (\%) and (d) semen pH (with reference line at $\mathrm{pH}=7$ ). 
Table 1: Mean \pm S.E.M., semen quality parameters between the first and last ejaculates after semen collection from Asian elephant bulls $(\mathrm{N}=8)$ on alternate days for ten days.

\begin{tabular}{|c|c|c|c|c|c|}
\hline \multirow{2}{*}{ Parameter } & \multicolumn{2}{|c|}{ Ejaculate } & \multirow[b]{2}{*}{$\mathrm{t}$} & \multirow[b]{2}{*}{$\mathrm{df}$} & \multirow[b]{2}{*}{$p$-value } \\
\hline & First & Last & & & \\
\hline Ejaculate volume (mL) & $35.8 \pm 14.5$ & $17.6 \pm 8.7$ & 1.07 & 7 & 0.318 \\
\hline Sperm concentration $\left(\times 10^{6}\right.$ sperm $\left./ \mathrm{mL}\right)$ & $1593.0 \pm 416.4$ & $1065.9 \pm 221.0$ & 1.30 & 7 & 0.235 \\
\hline Semen pH & $7.1 \pm 0.4$ & $7.8 \pm 0.3$ & -2.43 & 7 & $0.045^{\star \star}$ \\
\hline Motile spermatozoa $(\%)^{*}$ & $1.5 \pm 1.2$ & $11.5 \pm 7.3$ & -1.46 & 7 & 0.187 \\
\hline Membrane intact spermatozoa $(\%)^{*}$ & $13.8 \pm 6.5$ & $23.9 \pm 8.8$ & -1.35 & 7 & 0.221 \\
\hline Spermatozoa with detached head (\%) & $73.8 \pm 7.3$ & $52.3 \pm 7.8$ & 2.80 & 7 & $0.026^{\star \star}$ \\
\hline Spermatozoa with attached head (\%) & $26.3 \pm 7.3$ & $47.8 \pm 7.8$ & -2.80 & 7 & $0.026^{\star \star}$ \\
\hline \multicolumn{6}{|l|}{ Spermatozoa with attached head } \\
\hline Head abnormality $(\%)^{*}$ & $11.9 \pm 6.5$ & $5.0 \pm 3.2$ & 3.43 & 7 & $0.011^{* *}$ \\
\hline Mid-piece abnormality (\%) & $12.1 \pm 3.1$ & $7.1 \pm 2.5$ & 2.69 & 7 & $0.031^{\star *}$ \\
\hline Tail abnormality $(\%)^{*}$ & $17.4 \pm 7.3$ & $12.3 \pm 2.3$ & 0.27 & 7 & 0.793 \\
\hline Normal morphology (\%) & $58.6 \pm 10.5$ & $75.6 \pm 4.5$ & -2.13 & 7 & 0.071 \\
\hline Total spermatozoa with normal morphology (\%) & $16.5 \pm 6.3$ & $37.3 \pm 6.6$ & -3.73 & 7 & $0.007^{\star *}$ \\
\hline
\end{tabular}

*Percentages of motile, membrane intact spermatozoa and percentage of intact spermatozoa with head abnormalities were square root transformed before being analyzed with the paired t-tests. ${ }^{* *}$ Significant differences between first and last ejaculate at $p<0.05$. 
Table 2: The semen quality and ampullary diameter* at the day of transrectal ultrasonographic assessment of Asian elephant bulls $(\mathrm{N}=6)$ in experiment 2. Bulls 1 and 2 have been used as breeding bulls at the National Elephant Institute, Thailand and were assumed to represent bulls with acceptable semen quality. Bulls A2, A3, B4 and B5 used in experiment 1 were regarded as representative of bulls with poor semen quality.

\begin{tabular}{lcccccc}
\hline Parameter & Bull 1 & Bull 2 & A2 & A3 & B4 & B5 \\
\hline Age & 32 & 42 & 54 & 59 & 21 & 22 \\
Ejaculate volume (mL) & 9 & 3 & 30 & 52 & 11 & 30 \\
Sperm concentration (x 106 sperm/mL) & 335 & 550 & 910 & 1650 & 181 & 1370 \\
Semen pH & 8 & 7.98 & 5.52 & 6.46 & 8.54 & 7.55 \\
Motile spermatozoa (\%) & 80 & 40 & $<5$ & 0 & $<5$ & $<5$ \\
Membrane intact spermatozoa (\%) & 82 & 65 & 6.5 & 5.5 & 23.5 & 46.5 \\
Spermatozoa with detached head (\%) & 5 & 28 & 65 & 82 & 26 & 2 \\
Spermatozoa with attached head (\%) & 95 & 72 & 35 & 18 & 74 & 98 \\
Attached head spermatozoa with & & & & & & \\
$\quad$ Head abnormality (\%) & 0 & 0 & 0 & 3 & 28 & 0 \\
$\quad$ Mid-piece and tail abnormalities (\%) & 15 & 18 & 51 & 70 & 21.5 & 14.5 \\
$\quad$ Normal morphology (\%) & 85 & 82 & 49 & 27 & 50.5 & 85.5 \\
Total spermatozoa with normal morphology (\%) & 80.8 & 59.0 & 17.2 & 4.9 & 37.4 & 83.8 \\
Left ampullar diameter (cm) & 5.50 & 4.31 & 2.96 & 1.59 & 2.12 & 5.35 \\
Right ampullar diameter (cm) & 4.48 & 3.13 & 2.39 & 3.50 & 2.59 & 3.78 \\
\hline
\end{tabular}

Ampullar diameter refers to the diameter of the ampullary region of the vas deferens. 

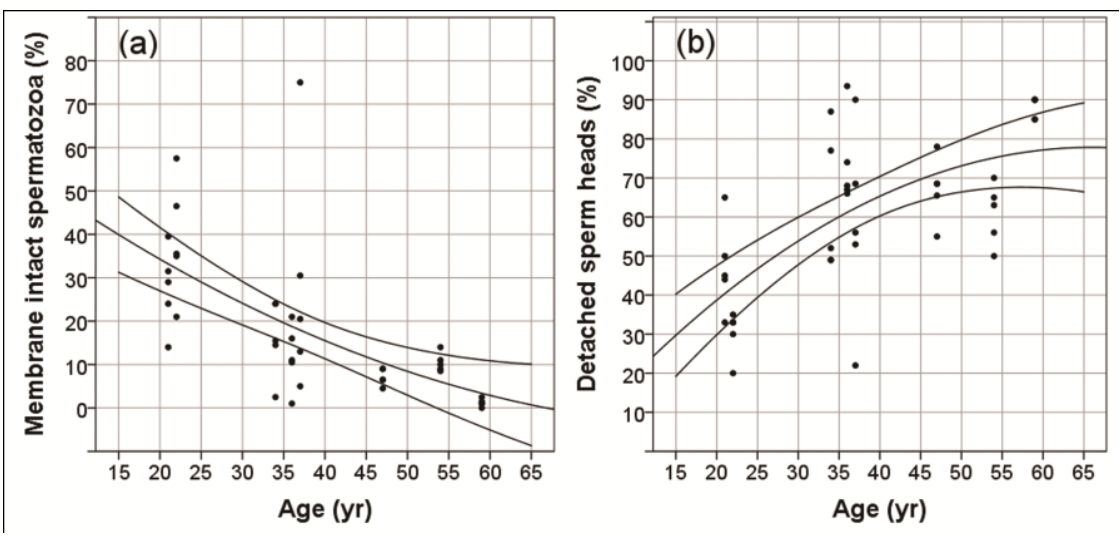

Figure 2: Scatter plots showing the relationship between age and semen quality in mature Asian elephant bulls ( $n=40,8$ bulls $\times 5$ ejaculates); (a) sperm membrane integrity (\%) and (b) detached sperm heads (\%).

\section{Experiment 2: Effect of ampullar diameter}

The individual values for semen quality and diameter of both the left and right ampullae for the six bulls are shown in table 2 . The diameter of the ampullae correlated positively with the percentages of motile $(r=0.616, p<0.05)$, morphologically normal $(r=0.749$, $p<0.05)$ and membrane intact spermatozoa $(r=0.735, p<0.01)$. The ampullary diameter was negatively correlated with the percentage of intact (attached head) spermatozoa with head abnormalities $(r=-0.619, p<0.05)$ and the percentage of spermatozoa with a detached head $(r=-0.622, p<0.01)$ (Figure 3$)$. No correlation between the diameter of the ampullae and the age of the bulls was evident.
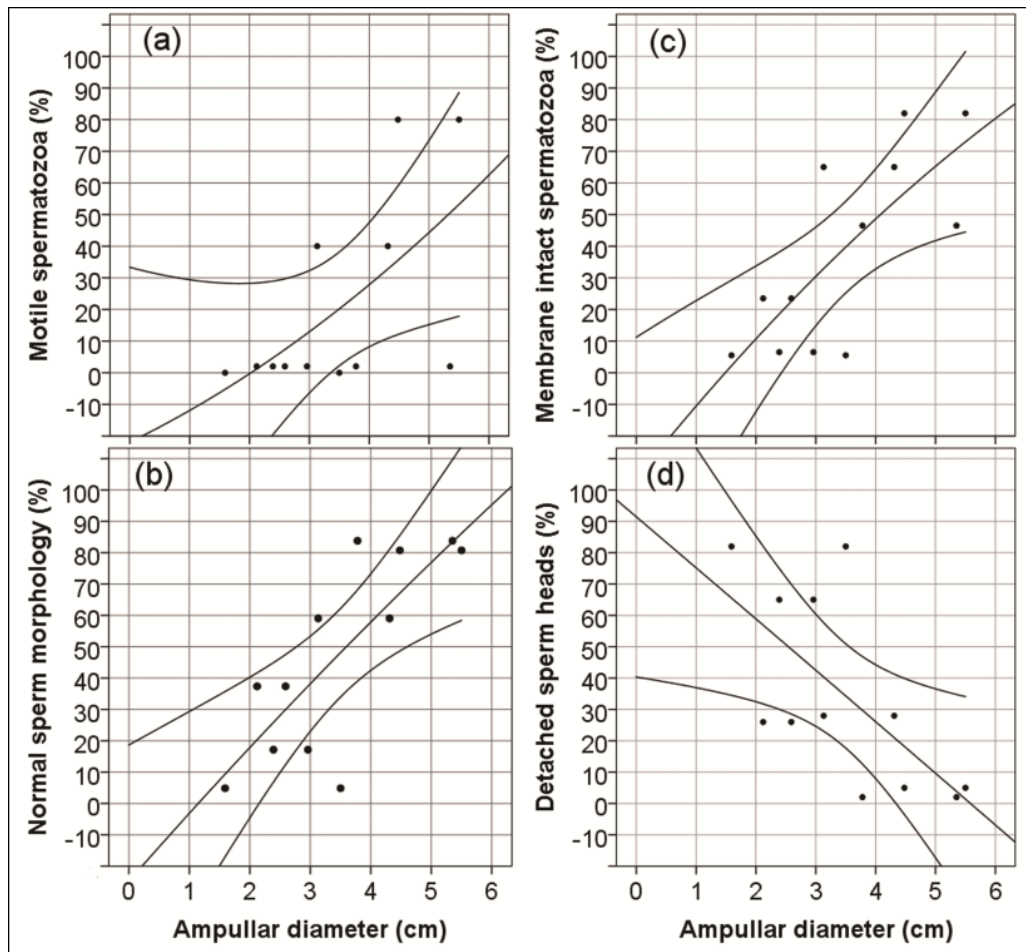

Figure 3: Scatter plots showing the relationship between ampullar diameter and semen quality in mature Asian elephant bulls $(n=12 ;, 6$ bulls, each with a pair of ampullae); (a) sperm motility (\%), (b) normal sperm morphology (\%), (c) sperm membrane integrity (\%) and (d) detached sperm heads (\%). 


\section{Discussion}

The bulls selected for this study on the basis of previous poor semen quality, and a prolonged period of sexual rest, seemed to have senescent spermatozoa in their reproductive tract, given that they demonstrated high proportions of dead spermatozoa and spermatozoa with detached heads in initial ejaculates, as described for other species (Barth, 2007). The alternate day semen collection regimen resulted in improved sperm morphology from the first to the last ejaculate; moreover, the incidence of sperm with detached heads was reduced by the end of the collection period. Furthermore, the percentages of motile and membrane intact sperm were greater after repeated semen collection. Although the changes in sperm motility and membrane integrity were not statistically significant when tested across the whole group of bulls, the effect was bull-dependent and was dramatic in one individual where the percentages of motile spermatozoa improved from $0 \%$ to $60 \%$, and those of membrane intact spermatozoa from $5 \%$ to $75 \%$, while the proportion of spermatozoa with detached heads decreased from $90 \%$ to $22 \%$. These effects were similar to those described in cattle, where improved quality of spermatozoa and subsequent reproductive performance have been reported following frequent repeated semen collection for domestic bulls diagnosed with sperm accumulation (Barth, 2007). Besides the improvement of aspects of semen quality, semen $\mathrm{pH}$ changed from neutral to alkaline. The alkalinity of semen relates not only to the exact combination of prostatic (acidic) and seminal vesicular (alkaline) fluids within the seminal plasma but can also be affected by urine contamination (alkaline), which is quite common following semen collection assisted by transrectal massage (Imrat et al., 2012a). Indeed, many aspects of semen composition, including volume and $\mathrm{pH}$, vary between individual bulls and ejaculates following collection by transrectal massage (Imrat et al., 2013).

Male elephants reach sexual maturity / puberty and develop the ability to produce normal spermatozoa somewhere around $10-15 \mathrm{y}$ of age; however, they only become capable of mating female elephants when they are about $18-20 \mathrm{y}$ and are physically large enough to mount an adult female (Schulte, 2006). Since, an Asian elephant's expected lifespan is approximately $60-70$ y (Choudhury et al., 2008), the bulls regarded as producing poor quality semen in this study spanned the age ranges of 
young mature adult to reproductively aged $(21-59 \mathrm{y})$. The lower percentages of membrane intact spermatozoa associated with increasing age are similar to the results of a previous study (Thongtip et al., 2008a) where the oldest group (51 - $70 \mathrm{y}$ ) of Asian elephant bulls had the lowest percentages of membrane intact spermatozoa. Similar correlations between semen quality and age have been observed in other species. In man, male aging also has an effect on fertility and semen quality parameters (Kidd et al., 2001). The semen quality of men starts to decrease from a mean age of $40 \mathrm{y}$ (Guerrero et al., 2008). On the other hand, older male African elephants (45-60 y) in wild populations father more calves than younger males. The explanation for the higher reproductive success of older bulls in the wild is thought to be related primarily to a longer duration of musth in the dominant males, and the related finding that $74 \%$ of calves are sired by male elephants in musth (Hollister-Smith et al., 2007). In captive Asian elephants, however, it is not known how semen quality or fertility of bulls in musth compares to that of non-musth bulls quite simply because bulls in musth are too aggressive to safely approach and use for breeding or semen collection. In the current study, increasing age was associated with a higher percentage of sperm with detached heads. Similarly, in other species older males have been reported to exhibit higher rates of sperm accumulation and senescence than younger males (Pizzari et al., 2008). In the present study, the mean percentage of membrane intact sperm was below $10 \%$, and the percentage with detached heads was over $80 \%$ for bulls aged 35 $y$ and above. The underlying cause of the increased incidence of detached sperm heads is not, however, clear. Other than sperm accumulation or aging of the animal, inflammation and infection of the seminal vesicles, ampullae and/or epididymides have also been associated with increased incidences of detached sperm heads (Nothling and Volkmann, 1997).

As in the current study, the maximum ampullary diameter in male Asian and African elephants when evaluated by ultrasound has been reported to be $5 \mathrm{~cm}$ (Hildebrandt et al., 2000). Slightly unexpectedly, a larger diameter of the ampullae in the current study was associated with improvements in semen quality, such as increased percentages of motile, membrane intact and morphologically normal spermatozoa and reduced percentages of spermatozoa with detached heads; this contrasts with the high incidence of detached sperm heads and low semen quality in bulls and stallions with enlarged and dilated ampullae as a result of the accumulation of spermatozoa 
(Nothling and Volkmann, 1997). The function of the accessory sex glands and epididymides may be negatively affected by decreasing serum testosterone concentrations as a result of aging (Thongtip et al., 2008a). One previously reported change is a narrowing of the diameter of the ampullary lumen (Elzanaty, 2007). In a previous study the oldest (51-70 y) elephants presented the lowest serum and seminal plasma testosterone concentrations (Thongtip et al., 2008a), but in this latter study the age of bulls did not correlate with ampullar diameter.

In summary, the accumulation of senescent spermatozoa appears to contribute to poor semen quality in some Asian elephant bulls, and can be improved by frequent (alternate day) semen collection; however, while this regimen led to clear improvements in one bull and reduced percentages of spermatozoa with detached heads in all, sperm membrane integrity and motility remained low in most animals suggesting either that there were additional factors contributing to poor sperm quality and/or that alternate day semen collection for $10 \mathrm{~d}$ was not sufficient to completely evacuate the senescent spermatozoa. Increasing age and narrow ampullar diameter also appear to negatively influence semen quality in Asian elephants, although the relationship between semen quality and ampullar diameter and function in this species requires further clarification.

\section{Acknowledgements}

The authors thank the EU-Asia-Link Project on "Managing the Health and Reproduction of Elephant Populations in Asia" TH/Asia Link/012 (9141055) for providing the funds for Podjana Imrat's PhD scholarship and the described studies. We also thank the staff at the National Elephant Institute (NEI), Lampang, Thailand for their support.

\section{References}

Bane, A., 1952. A study on the technique of hemocytometric determination of sperm motility and sperm concentration in bull semen. Cornell Vet 42, 518-531.

Barth, A.D., 2007. Sperm accumulation in the ampullae and cauda epididymides of bulls. Anim Reprod Sci 102, 238-246. 
Bjorndahl, L., Soderlund, I., Kvist, U., 2003. Evaluation of the one-step eosin-nigrosin staining technique for human sperm vitality assessment. Hum Reprod 18, 813-816.

Brown, J.L., 2000. Reproductive endocrine monitoring of elephants: an essential tool for assisting captive management. Zoo Biol 19, 347 - 367.

Choudhury, A., Lahiri Choudhury, D.K., Desai, A., Duckworth, J.W., Easa, P.S., Johnsingh, A.J.T., Fernando, P., Hedges, S., Gunawardena, M., Kurt, F., Karanth, U., Lister, A., Menon, V., Riddle, H., Rübel, A., Wikramanayake, E., 2008. Elephas maximus, In: IUCN 2012, editor. IUCN Red List of Threatened Species. Version 2012.2.

Elzanaty, S., 2007. Association between age and epididymal and accessory sex gland function and their relation to sperm motility. Arch Androl 53, 149-156.

Garner, D.L., Hafez, E.S.E., 2000. Spermatozoa and seminal plasma In: Hafez, E.S.E., Hafez, B. (Eds.), Reproduction in farm animals, Lippincott Williams and Wilkins, Baltimore, Maryland, p. 509 p.

Guerrero, C., Kendo-McAnlis, K., Kaufmann, R., 2008. Effect of age on semen quality of 16,156 patients attending an infertility clinic. Fertil Steril 90 Suppl. 1, S385-S386.

Hildebrandt, T.B., Hermes, R., Pratt, N.C., Fritsch, G., Blottner, S., Schmitt, D.L., Ratanakorn, P., Brown, J.L., Rietschel, W., Göritz, F., 2000. Ultrasonography of the urogenital tract in elephants (Loxodonta africana and Elephas maximus): an important tool for assessing male reproductive function. Zoo Biol 19, 333-345.

Hollister-Smith, J.A., Poole, J.H., Archie, E.A., Vance, E.A., Georgiadis, N.J., Moss, C.J., Alberts, S.C., 2007. Age, musth and paternity success in wild male African elephants, Loxodonta africana. Animal behaviour 74, 287-296.

Imrat, P., Hernandez, M., Rittem, S., Thongtip, N., Mahasawangkul, S., Gosalvez, J., Holt, W.V., 2012a. The dynamics of sperm DNA stability in Asian elephant (Elephas maximus) spermatozoa before and after cryopreservation. Theriogenology 77, 998-1007.

Imrat, P., Suthanmapinanth, P., Saikhun, K., Mahasawangkul, S., Sostaric, E., Sombutputorn, P., Jansittiwate, S., Thongtip, N., Pinyopummin, A., Colenbrander, B., Holt, W.V., Stout, T.A., 2013. Effect of pre-freeze semen quality, extender and cryoprotectant on the post-thaw quality of Asian elephant (Elephas maximus indicus) semen. Cryobiology 66, 52-59.

Kidd, S.A., Eskenazi, B., Wyrobek, A.J., 2001. Effects of male age on semen quality and fertility: a review of the literature. Fertil Steril 75, 237-248. 
Magistrini, M., 2000. Semen evaluation, In: Samper, J.C. (Ed.), Equine breeding management and artificial insemination, W.B. Saunders company, Philadelphia, Pennsylvania, pp. 91-108.

Nothling, J.O., Volkmann, D.H., 1997. Dilatation of the Ampullae and an Increased Incidence of Loose Sperm Heads after Bilateral Vesiculectomy in a Bull. Reprod Dom Anim 32, 321-324.

Parthasarathy, A., Nair, M.K.C., 2006. Sexual Reproductive Health of Young People (10-24 Years). Jaypee Bros. Medical Publishers, New Delhi, India.

Pizzari, T., Dean, R., Pacey, A., Moore, H., Bonsall, M.B., 2008. The evolutionary ecology of pre- and post-meiotic sperm senescence. Trends Ecol Evol 23, 131-140.

Pycock, J.F., 2008. Stallion reproduction, semen collection and artificial insemination, the 30th Bain Fallon Memorial Lectures: Dermatology, Reproduction, Dentistry and Evidence-Based Medicine, Equine Veterinarians Australia, Cairns, Queensland, Australia, pp. 407-440.

Saragusty, J., Hildebrandt, T.B., Behr, B., Knieriem, A., Kruse, J., Hermes, R., 2009. Successful cryopreservation of Asian elephant (Elephas maximus) spermatozoa. Anim Reprod Sci 115, 255-266.

Sarder, M.J.U., 2008. Effects of age, body weight, body condition and scrotal circumference on sperm abnormalities of bulls used for artificial insemination ( $\mathrm{Al}$ ) programme in Bangladesh. University Journal of Zoology Rajshahi University 2008 27, 73-78.

Schmitt, D.L., Hildebrandt, T.B., 1998. Manual collection and characterization of semen from Asian elephants (Elephas maximus). Anim Reprod Sci 53, 309-314.

Schmitt, D.L., Hildebrant, T.B., Hermes, R., Goritz, F., 2001. Assisted reproductive technology in elephants, Proc 1st Int Symp Assisted Reproductive Technology for Conservation Genetic Management of Wildlife, Omaha's Henry Doorly Zoo, pp. 15-17.

Schulte, B.A., 2006. Behavior and social life, In: Fowler, M.E., Mikota, S.K. (Eds.), Biology, Medicine, and Surgery of Elephants, Blackwell Publishing, Ames, lowa, pp. 35-43.

Thitaram, C., 2012. Breeding management of captive Asian elephant (Elephas maximus) in range countries and zoos. Jpn J Zoo Wildl Med 17, 91-96.

Thongtip, N., Mahasawangkul, S., Thitaram, C., Pongsopavijitr, P., Kornkaewrat, K., Pinyopummin, A., Angkawanish, T., Jansittiwate, S., Rungsri, R., Boonprasert, K., Wongkalasin, W., Homkong, P., Dejchaisri, S., Wajjwalkul, W., Saikhun, K., 2009. Successful artificial insemination in the Asian elephant (Elephas maximus) using chilled and frozenthawed semen. Reprod Biol Endocrin 7, 75-82. 
Thongtip, N., Saikhun, J., Mahasawangkul, S., Kornkaewrat, K., Pongsopavijitr, P., Songsasen, N., Pinyopummin, A., 2008a. Potential factors affecting semen quality in the Asian elephant (Elephas maximus). Reprod Biol Endocrinol 6, 9.

Thongtip, N., Saikhun, J., Mahasawangkul, S., Kornkaewrat, K., Suthanmapinanh, P., Pinyopummin, A., 2008b. Effect of Pentoxifylline on the Motility Characteristics and Viability of Spermatozoa in Asian Elephants (Elephas maximus) with Low Semen Quality. Thai J Vet Med $38,37-45$. 